Automated Detection and Resolution of Legal Cross References: Approach and A Study of Luxembourg's Legislation

By: Morayo Adedjouma, Mehrdad Sabetzadeh, Lionel Briand

Software Verification and Validation Laboratory

Interdisciplinary Centre for Security, Reliability and Trust (SnT)

University of Luxembourg

February 18, 2014 


\title{
Automated Detection and Resolution of Legal Cross References: Approach and A Study of Luxembourg's Legislation
}

\author{
Morayo Adedjouma, Mehrdad Sabetzadeh, Lionel Briand \\ SnT Centre for Security, Reliability and Trust \\ University of Luxembourg, Luxembourg \\ \{morayo.adedjouma, mehrdad.sabetzadeh, lionel.briand\}@uni.lu
}

\begin{abstract}
When identifying and elaborating compliance requirements, analysts often need to follow the cross references in the underlying legal texts and consider the additional information in the cited provisions. To enable easier navigation and handling of cross references, automation is necessary for recognizing the natural language patterns used in cross reference expressions (cross reference detection), and for interpreting these expressions and linking them to the target provisions (cross reference resolution). Numerous approaches have been developed over the years to automate the detection and resolution of cross references in legal texts; however, certain facets, e.g., the natural language patterns used in cross references, remain under-explored. Salient gaps also remain towards a systematic understanding of the cross reference resolution process. In this paper, we propose a flexible solution for automated detection and resolution of cross references in legal texts, aimed at addressing the observed gaps. We ground our work on Luxembourg's legislative texts, both for studying the natural language patterns in cross reference expressions and for evaluating the effectiveness of our solution.
\end{abstract}

Keywords-Legal Compliance, Cross References, Natural Language Processing

\section{INTRODUCTION}

Legal compliance is a key concern for many software applications. Information systems in domains such as government, healthcare and finance are, for example, subject to a host of laws and regulations aimed at protecting security and privacy. Systems used by governments for public administration further need to comply with public regulatory frameworks such as tax and social welfare laws. In all cases, non-compliance can have serious consequences, including fines, lawsuits, damage to public trust, loss of business, and even criminal prosecution.

To identify and elaborate compliance requirements, analysts need to review and analyze the relevant legal texts. An important complexity that arises in so doing is that legal provisions are interrelated and cannot be considered in isolation. The relationships between provisions in legal texts are captured using cross references. To illustrate, consider the excerpt in Fig. 1 from Article 2 of Luxembourg's Income Tax Law [1]:

Art. 2. [...] Individuals are considered non-resident taxpayers if they do not reside in Luxembourg but have a local income as per the definition of Art. 156.

Fig. 1. Using cross references for relating legal provisions

The excerpt (translated from French) has a cross reference to Art. 156. To understand what a non-resident taxpayer is, one needs knowledge of what local income is. This example shows only one usage of cross references, i.e., specifying a dependency for a definition - in this case, the definition of local income. Cross references may be used, among other reasons, for adding exceptions and constraints, specifying priority between provisions, and making amendments [2].

Many Requirements Engineering activities concerned with legal compliance need to account for cross references in legal texts. For example, Breaux et al. [3], [4] and Maxwell et al. [5], [6] follow cross references and analyze the cited provisions for identifying constraints, priorities, exceptions, refinements, and conflicts between compliance requirements; Ghanavati et al. [7] analyze cross referenced provisions while building models of compliance requirements.

To perform these activities in a more thorough and efficient manner, it is useful to have legal texts structured as markup documents, e.g., in an XML format, with cross references represented as (navigable) links [8], [9]. Doing so requires the ability to first recognize natural language expressions that denote cross references (cross reference detection), and then to interpret and link these expressions to the target provisions (cross reference resolution). The resulting links on the one hand enable easier and more structured exploration of legal texts by analysts, and on the other hand, provide a basis for further analysis, e.g., traceability and impact analysis. A typical legal text can contain hundreds and sometimes thousands of cross references. Automated support is thus necessary for cross reference detection and resolution.

The objective of this paper is to develop a flexible framework for automated detection and resolution of cross references in legal texts. Several approaches already exist for this purpose [10], [11], [9], [12], [13]; but, as we argue in more detail in Section III, important gaps remain. Most notably:

- Cross reference detection and resolution necessarily require knowledge of the internal structure of legal texts, i.e., how these texts are organized into sections, articles, paragraphs, and so on. Typically, an explicit schema is defined to characterize this structure [9], [8]. However, significant manual work is necessary to transform a non-markup legal text (e.g., in PDF or plain text format) into a markup document (e.g., in XML format) that conforms to the schema.

- In several countries, best practices exist on how to phrase and use cross references in legal texts. For example, in the 
US, the Bluebook [14] and the US Association of Legal Writing Directors' (ALWD) Citation Manual lay down specific conventions for cross references. These best practices, as already observed by others [12], are often inadequate for accurate detection of cross references, particularly in older legal corpora, e.g., public law. Grounded Theory studies of actual legal texts, e.g., as done by Breaux [9] and de Maat et al. [11], provide valuable insights about the flexible natural language patterns used for specifying cross references; however, further investigations of actual legal texts is required to understand commonalities between legal texts in different countries and developing reusable natural language patterns for cross reference detection.

- The majority of the existing work does not clearly distinguish cross reference detection and the more complex task of cross reference resolution. Consequently, important subtleties that arise during resolution have not been adequately addressed, e.g., disambiguation when the cross reference patterns are ambiguous.

To address these gaps, we make the following contributions:

1) We describe how a schema for the structure of legal texts can be used to automatically transform non-markup texts into texts with structural markup (Section V). This task is a prerequisite for cross reference resolution and, if done manually, is effort-intensive for large legal texts.

2) We report on a study of Luxembourg's Income Tax Law [1] aimed at identifying the natural language patterns used in this law for cross references (Section VII-A). This law, first drafted in 1967, is written in French and has a total of 1223 cross reference expressions. We conclude from this study that the resulting patterns closely resemble those identified in a similar study of the Dutch laws [11], lending support to these patterns being reusable across different legal jurisdictions. In addition, our study identifies natural and important variations of these patterns that were not previously seen.

3) We provide a systematic treatment of cross reference resolution addressing, in an algorithmic way, subtleties that one needs to take into account with regards to the interpretation of complex cross reference expressions (Sections VII-B). While our treatment is applicable to both intra- and inter-document cross references, only the former category (commonly referred to as internal cross references) is considered in this paper.

4) Building on a Natural Language Processing (NLP) platform, GATE [15], and an efficient first-order logic interpreter, Crocopat [16], we develop tool support for (1) transformation of non-markup legal texts into legal texts with structural markup, (2) automated detection and resolution of cross references, and (3) conducting cross reference analysis (Section IX).

5) We report on an empirical evaluation of our approach, arguing about the generalizability of the identified cross reference patterns, the quality of automated resolution, and the scalability of our approach (Section X).

The illustrative examples in the paper are real and drawn from Luxembourg's Income Tax Law. To improve readability, we use English translations while preserving the essence of the structure of the original cross reference expressions in French.

\section{BACKGROUND AND TERMINOLOGY}

A (legal) cross reference is a citation that links one legal provision to another [5]. We distinguish cross references from cross reference expressions. A cross reference expression is a Natural Language (NL) phrase that represents one or more cross references. For example, "Articles 30 and 102 of the law of April 29, 1964 concerning family benefits" is a cross reference expression. This expression embodies two cross references: one to "Article 30" and another to "Article 102" of the respective law. To avoid repetition, we abbreviate "cross reference" to $\boldsymbol{C R}$ and "cross reference expression" to $\boldsymbol{C R} \boldsymbol{E}$ in the rest of the paper. We note that, with the distinction made between CR and CRE, it would be more accurate to refer to CR detection and CR resolution as CRE detection and CRE resolution, respectively. We ignore this technicality when referring to detection and resolution activities.

When a CRE points to provisions within the same legal text as where the CRE appears, the CRE is called internal; otherwise, when a CRE points to provisions in a different legal text, it is called external [17]. In the example of Fig. 1, "Art. 156" is an internal CRE. The example in the previous paragraph (i.e., "Articles 30 and 102 of ...") is an external CRE.

CREs can be further classified as explicit, implicit, or delegating. If a CRE is defined in terms of the alphanumeric labels of legal text elements, it is called explicit. All our examples so far where articles were referred to by their numbers were explicit. In contrast, an implicit CRE uses some adjective, adverb, or anaphor to refer to the target provisions [11] , e.g., "this article" and "the following paragraphs". The third class, namely delegating, exclusively applies to external CREs. This class of CREs is used when a legislative text delegates authority to regulations for further elaboration. Regulations reside at a lower level than legislation in the hierarchy of legal texts and are usually subject to more frequent changes. Legislative texts seldom refer to regulations in a precise way and typically only indicate the nature of the regulations being cited. An example delegating CRE is "Grand-Ducal regulation" in the following: "A Grand-Ducal regulation shall provide the details for $[\ldots]$ ".

Finally and with regards to implicit CREs, there are occasions where legal texts use vague terms such as "provision" (in French: "disposition" or "prescription"), e.g., "the above provision". We refer to these CREs as unspecific. Unspecific CREs cannot be conclusively associated with specific structural elements, e.g., articles or paragraphs. They cannot thus be resolved with reasonable accuracy through automation. All CREs except for delegating and unspecific ones are in principle resolvable through automation.

\section{RELATED WORK}

Breaux et al. [3], [9] identify natural language patterns for CREs based on a study of 118 expressions across three US regulations. They propose the use of an explicit schema for modeling the structure of legal texts. Similar to this earlier work, we study actual legal texts for identifying CRE patterns 
and use an explicit model to characterize the structure of legal texts. We consider a single but complete legal text (Luxembourg's Income Tax Law) with a total of 1223 CREs. We observe new patterns, not seen in the US regulations considered. Our study covers patterns for external CREs, not considered in [3], [9]. We propose automation for text structure markup, which is a manual process in [9].

The closest study to ours in terms of CRE patterns is de Maat et al.'s study of Dutch laws [11]. The differences in language aside, the patterns we observe in our investigation of Luxembourgish laws (written in French) are closely aligned with those in Dutch laws. In this sense, our study serves as a confirmatory measure for the generalizability of previouslyobserved patterns. In addition, we identify natural and important variations of these patterns. The main contributions of our work over De Maat et al.'s are: First, they assume that legal texts are already in a markup format with adequate structure to be transformed into the markup format required by their approach (MetaLex [8]). Our approach, in contrast, does not require pre-existing markup. Second, and more importantly, de Maat et al. do not clearly distinguish detection and resolution activities. They do not elaborate the resolution process, nor do they address the effectiveness of resolution in their evaluation. We instead provide a detailed treatment of resolution and measure its effectiveness in our evaluation.

Palmirani et al. [10] define CRE patterns based one legal writing guidelines for the Italian legal corpora. They tackle only detection and not resolution. Their approach does not address the generation of markup documents and their patterns are insufficient for recognizing many of the patterns seen in our study and that of the Dutch laws discussed above [11].

Hamdaqa et al. [12] propose an approach for resolving external CRs and report on a case study of three US regulations involving 122 (external) CREs. They use finite state machines for defining CRE patterns, based on the recommendations of the Bluebook [14] and ALWD's Citation Manual [18]. Their patterns are limited first in that they apply only to external CREs and second in that they are exclusively based on best practices and thus insufficient for the richer citation styles used in actual legal texts. Hamdaqa et al. consider automated markup generation through manually-written regular expressions. Our approach provides a more thorough and flexible framework than theirs. Our patterns encompass both internal and external CREs, and further are based on studying actual legal text. Our approach is parameterized by a schema, which enables us to automatically derive the necessary regular expressions for text markup generation.

Tran et al. [13] apply machine learning for CR detection and resolution in Japanese legislative texts. Similar to them, we distinguish CR detection and resolution. However, our detection and resolution strategies are algorithmic and rule-based. Using machine learning can be advantageous in that it does not require an a priori specification of CRE patterns. However, Tran et al. do not consider advanced patterns with recursive structures or multiple layers similar to those identified and addressed in our study (Section VII-A). It is unknown how

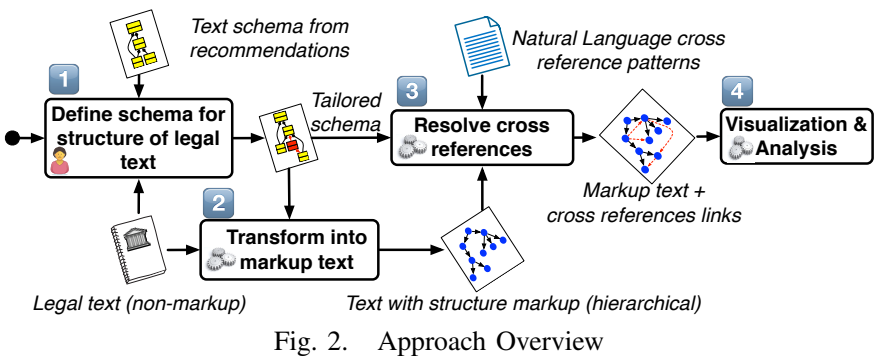

such patterns can be handled through learning. Further, for the patterns they do consider, they report an accuracy (F-measure) of $\approx 80 \%$ and $\approx 86 \%$ for detection and resolution respectively, with a combined accuracy of $67 \%$. This, compared to rulebased techniques, is low (see Section X).

\section{Approach OVERVIEW}

In this section, we provide an overview of our approach. The approach, shown in Fig. 2, has four main steps. The first step is manual and the remaining three steps are automatic.

Step 1 is concerned with defining a schema (metamodel) for expressing how a legal text is organized into subparts, e.g., books, chapters, articles, paragraphs and so on. In many jurisdictions, legal writing guidelines prescribe a generic and unified schema for structuring legal texts. Actual legal texts may nonetheless be only partially aligned with the generic schema. Some tailoring of the generic schema may thus be necessary in order to adapt it to a specific set of legal texts. The aim of Step 1, discussed in Section V, is to do this tailoring.

Step 2, discussed in Section VI, is concerned with transforming a non-markup text (e.g., in plain text or PDF format) into a markup text (e.g., in XML format). The transformation rules are automatically derived from the schema of Step 1.

The main step of the approach is Step 3. This step, discussed in Section VII, addresses the detection and resolution of CREs. Prerequisite to this step is knowledge of the NL patterns used in the CREs. These patterns, combined with the schema from Step 1, provide the syntactic and semantic basis for the CREs. As we argued earlier, the NL patterns used in actual legal texts can be richer and more versatile than what is prescribed in best practices. This necessitates examining actual legal texts for more accurate identification of the patterns.

Step 4, discussed in Section VIII, is concerned with using the outcomes of resolution for visualization and analysis.

\section{Defining a Legal TeXt Schema}

A text schema defines the grouping concepts, e.g., Chapter, Section and Article, used for organizing the content of a legal text. Having a text schema is essential for CR detection and resolution as both the syntax and interpretation of CRs depend on the underlying schema. We define a text schema via a UML class diagram. The classes in the diagram represent the grouping concepts used in a legal text. These classes are linked via aggregation associations representing the containment relationships between the concepts.

Fig. 3 shows the text schema for Luxembourg's Income Tax Law. To build this schema, we first developed a generic schema based on the legal writing guidelines published by the 


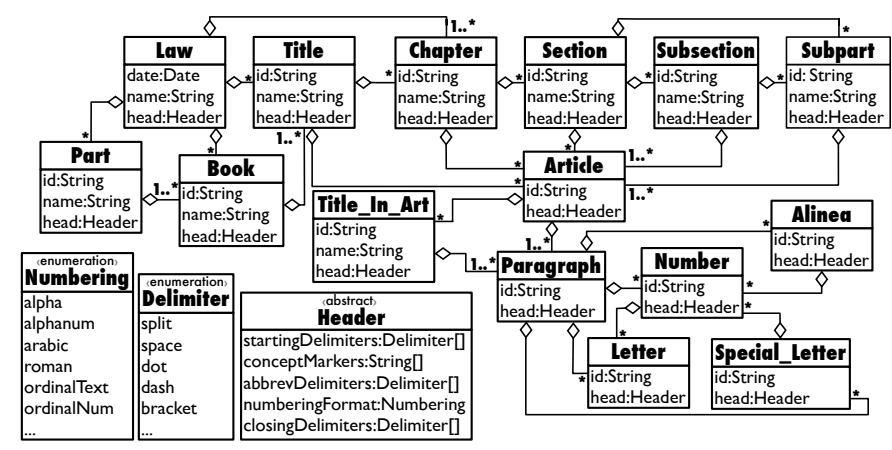

Fig. 3. Text schema for Luxembourg's Income Tax Law

State Council of Luxembourg [19]. The resulting schema was then discussed with legal experts and enhanced with additional grouping concepts specific to the tax legislation.

An individual legal text constitutes a Law. A Law may be hierarchically organized into Parts, Books, Titles, Chapters, Sections, and so on. Many levels in the hierarchy are optional, e.g., if Part is not used, one can go directly from Law to Book, Title, or Chapter. These (mutually exclusive) alternatives are captured in the model of Fig. 3 through different aggregation paths. The core grouping concept in a Luxembourgish legal text is that of an Article. The most common groups used under an article are Paragraphs and Alineas, and at a lower level Numbers, and Letters. Certain other nuanced groups, e.g., Title_In_Art, can be used in certain cases within articles.

Each grouping concept has a header (explained below) and an id. There is only one instance of Law per text, so the id is implicit in this case. Nevertheless, each law is associated with a publication date that needs to be captured. Some grouping concepts, e.g., chapters, have both an id and a name (chapter title). The header of a grouping concept $C$, called $C$ Header, provides information as to how the beginning of an instance

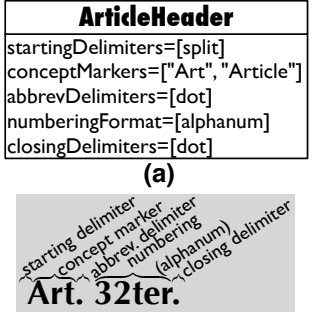

(b)

Fig. 4. (a) Header class for articles (b) Example article head of $C$ is recognized in the text. In the model of Fig. 3, only the abstract header class is shown. Each grouping concept defines a (static) specialization of this abstract class. As an example, we show ArticleHeader in Fig. 4. Here, the starting delimiter is a split (carriage return or linefeed), and the concept marker is "Art" or "Article". When the abbreviated concept marker is used, it may be (optionally) followed by a dot. The numbering is alphanumeric representing the identifier of the article in the legal text. The article header closes with a dot.

\section{Transforming Non-Markup to Markup TeXt}

We automatically derive from a text schema, e.g., the one in Fig. 3, regular expressions that transform non-markup legal texts to texts with structural markup. The automation builds on a simple observation: the natural structure of a textual document is such that a particular segment of text terminates only when we see a new grouping concept that is either at the same level as the current segment or at a level above the current segment. For example, assume that we have a document structured according to the schema of Fig. 3, and suppose that we are within a particular section, say Section 3. For this section to terminate, we either have to reach the beginning of Section 4, or, if there are no further sections, the beginning of a new chapter, a new title, etc.

The containment relationships between grouping concepts is never recursive. This means that we cannot have a grouping concept, say Chapter, which logically contains another grouping concept, say Section, and at the same time have Sections that contain Chapters. More precisely, a text schema, when viewed as a graph, is always an Acyclic Direct Graph (DAG). This in turn means that there is always some ordering of the grouping concepts, known as a topological ordering [20], that respects the containment relationships between the concepts. Computing this ordering is inexpensive and linear in the size of the input DAG [20]. One topological ordering for the schema of Fig. 3 in an ascending order is: [Letter, Number, Special_Letter, Dash, Alinea, Paragraph, Title_In_Art, Article, Subpart, Subsection, Section, Chapter, Title, Book, Part, Law]. Equipped with this ordering and the information from the CHeader classes in the text schema (Fig. 4), one can automatically generate the regular expressions that recognize the hierarchical structure of a legal text. The algorithm for generating and executing these regular expressions is shown in Algorithm 1.

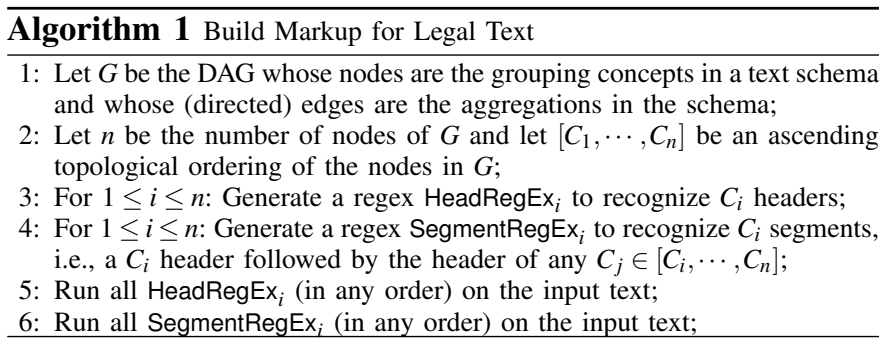

We illustrate the regular expressions for header identification (HeadRegEx) and segmentation (SegmentRegEx) over the Article grouping concept. Generating the regular expression for marking the head of articles (line 3 of the algorithm) is straightforward based on the information in the ArticleHeader class (Fig. 4). In Fig. 5(a), we show a regular expression, named MarkArticleHead and written in GATE's regular expression language [15], for marking article heads. Intuitively and in line with our previous discussion about ArticleHeader, MarkArticleHead looks for the following sequence: a split, an admissible concept marker, optionally a dot, an alphanumeric number, and a final dot. Note that at the time this regular expression is run, the text has already undergone lexical analysis with information about its tokens available.

Fig. 5(b) shows a regular expression, MarkArticleSegment, for marking article segments. This expression, also written in GATE's regular expression langauge, recognizes and annotates the text between the head of a given article and the head of the next grouping concept instance that is not logically containable in that article. From the topological ordering, we know which grouping concepts reside above Article and thus cannot be contained in articles. The segment for an article starts when its head is detected. The segment ends once the head of the 
immediately following article or of a higher level grouping concept, e.g., a chapter or a book, is detected. In the regular expression, there is a special EOD (End Of Document) level which has the largest topological order of all, meaning that $E O D$ terminates any segment at any other level.

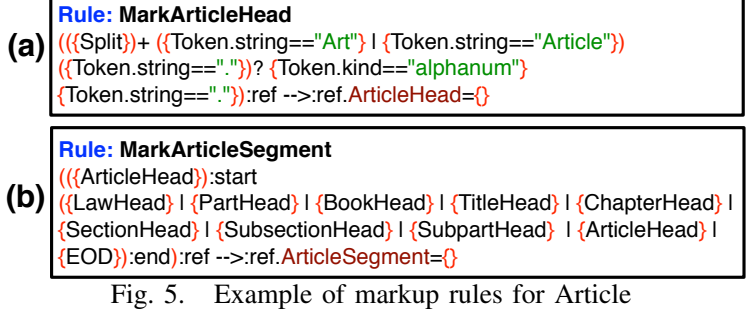

Due to space reasons, we cannot present all the technical details for generating these regular expressions. For example, groups such as chapters that have both ids and names have slightly more complex regular expressions that those for articles which only have ids. The annotations produced over a non-markup legal text by the regular expressions can be easily turned into a markup format, e.g., XML. The resulting markup text is the basis for the resolution process (Section VII).

\section{Detecting and Resolving CRoss References}

We break this step into three sequential sub-steps. The first sub-step addresses detection, and the second and third substeps address resolution. In the first sub-step, we automatically detect the CREs in a given legal text based on a set of predefined NL patterns (Section VII-A). In the second sub-step, the identified CREs are interpreted into a set of individual CRs (Section VII-B). Finally, in the third sub-step the individual CRs are linked to the target provisions (Section VII-C).

\section{A. NL Patterns for Cross References Expressions}

Our NL patterns were derived from a study of (Luxembourg's) Income Tax Law [1]. The current edition of this law, from January 2013, is 189 pages long and organized into 3 titles that collectively contain 15 chapters. The law has 236 articles, composed of 767 paragraphs. The large size and complexity of this law provides a rich basis for our investigation. Basing our work on the Income Tax Law was further motivated by our access to legal experts who could help us with understanding the structure and the content of this law. We analyzed all the 1223 CREs in the Income Tax Law and developed regular expressions to formalize the patterns we observed. The patterns, shown in Fig. 6, are organized into simple and complex. Complex patterns build on top of simple patterns, providing certain advanced features which we discuss over the course of this section.

We explain and illustrate our patterns, drawing on the terminology defined in Section II. We have made minor simplifications to the patterns for better readability. While the patterns were developed over French text, they carry over to English almost verbatim. With regards to the French grammar, there is only one simplification to note: In French, ordinals can appear both before and after nouns (e.g., "paragraphe premier", "premier paragraphe"); whereas in English, they can appear only

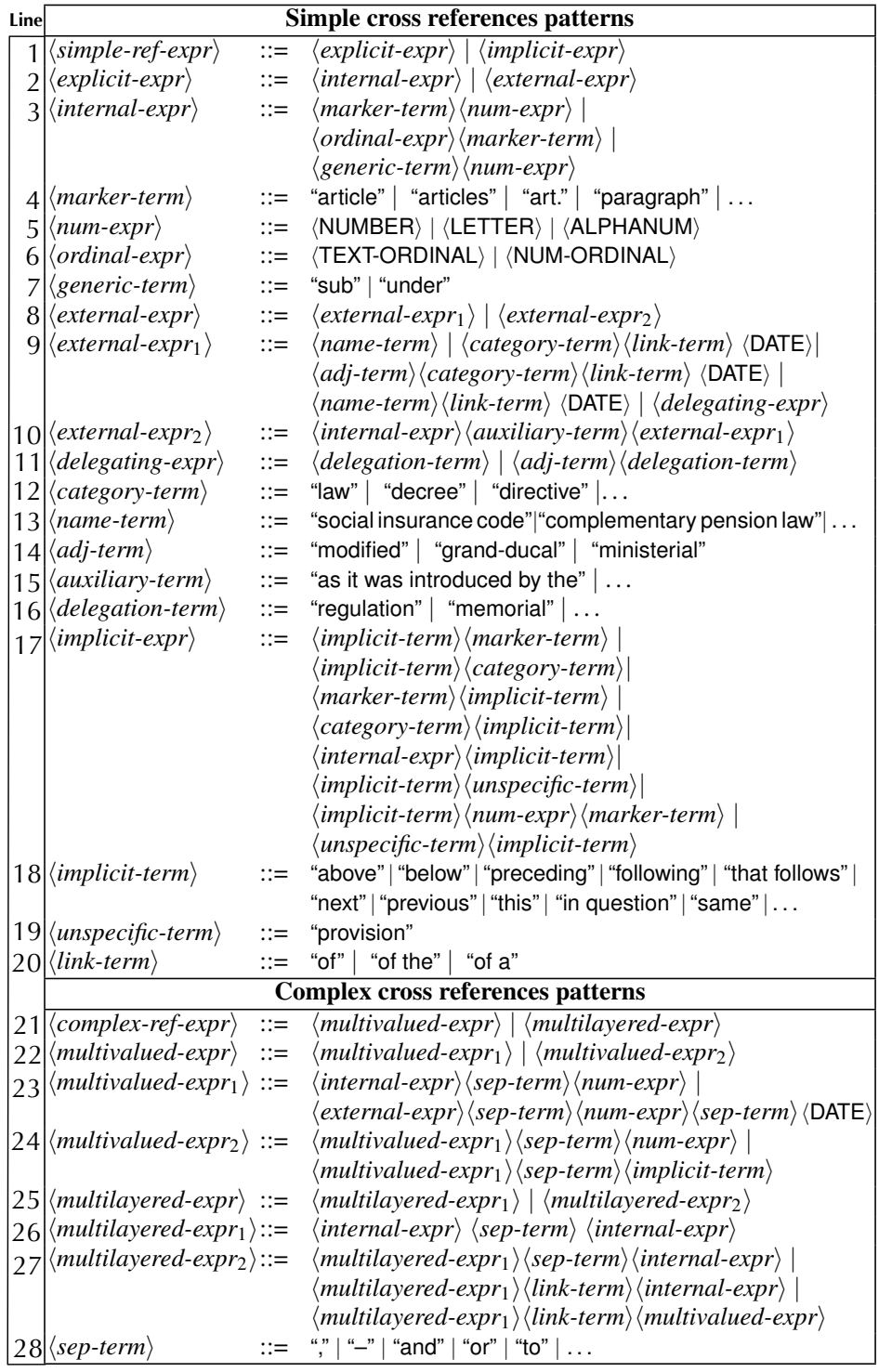

Fig. 6. Grammar for NL cross reference patterns.

before (e.g., "first paragraph"). In Fig. 6, symbols in upper-case letters, e.g., 〈NUMBER〉 denote terminals as identified by an NL lexical analyzer. Non-terminals that end with term, e.g., $\langle$ marker-term $\rangle$ and $\langle$ name-term $\rangle$ denote elements in predefined dictionaries (commonly known as gazetteers [15] in NLP). These terms vary from one legal jurisdiction and language to another and must be specified for a specific context.

Simple CREs. A simple CRE can be explicit or implicit (L. 1 of Fig. 6). Among explicit CREs, we distinguish internal and external (L. 2). Non-terminals 〈internal-expr〉 (L. 3) and $\langle$ external-expr (L. 8) respectively capture explicit internal and explicit external CREs.

An (explicit) internal CRE (L. 3-7) is either a concept marker, e.g., article, followed by a numerical expression, or an ordinal expression followed by a concept marker. The numerical expression can be an arabic number ("article 1"), a roman number ("chapter IV"), an alphanumeric ("alinea 2bis"), a number written out in text ("alinea four"), or a letter ("letter a"). A numerical expression may optionally have brackets around 
or at the end of it ("paragraph (2)", "paragraph 2)"). An ordinal expression can be a numerical ordinal ("1st article") or a textual ordinal ("first article").

A new pattern observed in our study and not reported in previous work is the case where a generic term, e.g., under, replaces a concept marker, e.g., letter. For example, "under a)" may be used in an article instead of "letter a)".

An (explicit) external CRE (L. 8-16) can be as simple as just the name of an external law, e.g., "social insurance code". Alternatively, an external CRE may be a phrase starting with an optional auxiliary term (e.g., "modified") followed by a legal text category and a date, e.g., "modified law of 23 July 1993". It is further possible for an external CRE to point to the internal provisions of an external law, e.g., "article 54bis as it was introduced by the Law of 23 July 1983". Delegating references (see Section II) also fall under external CREs.

A simple CRE may be implicit (L. 17-19), e.g., "this article". Among implicit CREs, there are some that cannot be resolved in an accurate way because they use an unspecific term, e.g., "the following provisions". An interesting variation of implicit CREs not previously reported are those that combine implicit terms and numerical expressions, e.g., "first four alineas".

Complex CREs. Complex CREs build on simple CREs, providing three additional features: enumerations, ranges, and navigation through legal text grouping concepts. Our overall classification of complex CREs follows de Maat et al.'s [11]: multivalued and multilayered (L. 21). Multilayered CREs can have multivalued parts (L. 27).

A multivalued CRE (L. 22-24) cites many provisions in the same expression by specifying only once a concept marker followed by a numerical expression. The numerical expression may be (1) an AND/OR enumeration, e.g., "numbers 1, 1a, 2 and 3" and "articles 22bis or 102"; (2) a range, e.g., "numbers 1 to 3 "; or (3) a combination of enumerations and ranges, e.g., "articles 119 to 121 and 124". Similar to simple CREs, multivalued CREs can use different numbering formats, e.g., ordinals as in "second and third alineas". Our grammar allows the repetition of enumerations and ranges within a CRE to accommodate cases seen in our study, e.g., "articles $144,147,148$ to 150,158 to $160,161,162$, and 163 ". We further allow multivalued CREs to include implicit terms, e.g., "articles 26-2, 27 and the following". Neither of these featurs are captured by de Maat et al. [11].

A multilayered CRE (L. 25-27) describes a navigation path through the hierarchy of a legal text. The navigation may be from an upper to a lower level, e.g., "article 91, 1st alinea, No 2". Alternatively, the navigation can be from a lower to an upper level, e.g., "second sentence of article 10 of the law of 23 may 1964". Finally, a navigation can be mixed-mode. That is, a CRE may start at a convenient hierarchical level, navigate upward or downward in the hierarchy, and then go in the reverse direction. For example, consider the following CREs: "article 3, paragraph (2) of the Law of 8 June 1999" and "numbers 3 and 4 of article 22bis, alinea 2". The navigation in the former is Article $\rightarrow$ Paragraph $\rightarrow$ Law and in the latter Number $\rightarrow$ Article $\rightarrow$ Alinea. Multilayered CRE may further use multivalued CREs in their makeup, e.g., "articles 59, alinea
3, 59bis, alinea 1, 170, alineas 2 and 3, 170bis, alineas 1 et 2, 170ter, alineas 1 and 2, and 172, alineas 4 and 5".

In Table I, we provide a breakdown of the 1223 CREs in the Income Tax Law accross three different dimensions: (1) Internal vs. External: 928 of the CREs are internal, of which 45 are unspecific, and 295 are external, of which 169 are delegating; (2) Explicit vs. Implicit: 839 of the CREs are explicit and the remaining 384 are implicit; and (3) Simple vs. Complex: 709 of the CREs are simple
TABLE I CRES IN INCOME TAX LAW

\begin{tabular}{|l|c|}
\hline CRE Type & \# of CREs \\
\hline Internal & 928 \\
- Unspecific & 45 \\
External & 295 \\
- Delegating & 169 \\
\hline Explicit & 839 \\
Implicit & 384 \\
\hline Simple & 706 \\
Complex & 517 \\
- Mutlivalued & 192 \\
- Multilayered & 325 \\
\hline
\end{tabular}
and the remaing 519 are complex. Among the complex ones, 192 are multivalued and 325 are multilayered.

We next present our approach for interpreting CREs into individual CRs.

\section{B. Interpreting Cross Reference Expressions}

The aim of the interpretation step is to translate each CRE into a set of individual CRs. The main complexity arising during interpretation is that some of the NL patterns in Section VII-A are ambiguous, i.e., several parse trees may exist for the same CRE. While a regular expression recognizer can delineate the start and end of each CRE even when the grammar is ambiguous, without knowing the structural makeup of the underlying legal text, one cannot choose the parse tree that is suitable for the text. Parser generators such as Yacc [21] require static priorities to be defined in order to resolve ambiguities. This is inadequate for CREs, because the admissible parse tree depends on the context, i.e., the actual legal text under analysis. Custom interpretation rules are thus necessary, as we detail in this section. To remain concise in our descriptions, we assume that the legal text under analysis has been already preprocessed. In particular, we assume that (1) ordinals, roman numbers, and numbers spelled out in text have been replaced with arabic numerals; alphanumerics remain unchanged; and, (2) abbreviated concept markers (e.g., art.) have been replaced with full labels (e.g., article).

\begin{tabular}{|c|c|}
\hline $\begin{array}{l}\text { Interpretting Simple CREs. } \\
\text { Among simple CREs, only } \\
\text { implicit ones and those using } \\
\text { generic terms (e.g., sub) need } \\
\text { treatment. We distinguish two } \\
\text { cases for implicit CREs: (1) } \\
\text { The ones that are semantically } \\
\text { equivalent to current, previous, }\end{array}$ & $\begin{array}{l}\text { Example } 1 \\
\text { CRE: current article } \\
\text { Context: article } 4 \text { paragraph } 2 \\
\text { Interp.: article } 4 \\
\text { Example } 2 \\
\text { CRE: following paragraphs } \\
\text { Context: article 122 paragraph } 1 \\
\text { Interp.: paragraph 2, paragraph } \\
\text { 2a, paragraph 3, paragraph } 4 \\
\text { Example } 3 \\
\text { CRE: same law } \\
\text { Prev. CRE: law of } 8 \text { june } 1999 \\
\text { Interp.: law of } 8 \text { june } 1999\end{array}$ \\
\hline
\end{tabular}
marker: In the case of current, the CRE is interpreted as pointing to the segment of the same type as concept marker that contains the CRE (Example 1). In the case of previous and next, the CRE is interpreted as pointing to segment(s) of the same type as concept marker that precede or succeed the 
CRE, respectively (Example 2). (2) Implicit CREs that are semantically equivalent to same or this followed by a concept marker. The interpretation of such a CRE is equivalent to that of the CRE coming immediately before it (Example 3).

Interpreting generic terms such as sub needs to be done according to the conventions in the legal jurisdiction

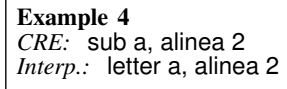

Interp.: letter a, alinea 2 to which the text belongs. In Luxembourg's legislation, the specific concept marker for a generic term can be inferred based on what is seen after the generic term. If the generic term is followed by a letter, the appropriate concept marker is Letter; otherwise, the concept marker is Number (Example 4).

\section{Interpreting multivalued CREs.}

A multivalued CRE that uses an enumeration is interpreted with the concept marker added to each element of the enumeration (Example 5). For interpreting CREs involving ranges, we distinguish grouping concepts that have unique numbering across an entire legal text (e.g., Article) and grouping concepts (e.g., Chapter, Paragraph) whose numbering is reset when a

Example 5
CRE: articles 14, 61, 91 ou 95
Interp.: article 14, article 61, article
91, article 95
Example 6
CRE: articles 99ter to 102
Legal Text: Lux. Income Tax Law
Interp.: article 99ter, article
99quater, article 100, article 101,
article 102
Example 7
CRE: paragraphs 1 to 3
Context: article 50bis, paragraph 4
Parent context: article 50bis
Interp.: paragraph 1, paragraph 2,
paragraph 3 interpretation attempt
Note: First inte
in the context of article 50bis,
paragraph 4 fails. Second attempt at
the level of article 50bis succeeds.
higher-level grouping concept is seen. For the former, we browse the entire hierarchical structure of the legal text to identify the elements in the range (Example 6). For the latter (grouping concepts whose numbering is reset), the interpretation depends on the context. We first attempt to interpret the CRE within the innermost segment in the hierarchy where the CRE appears. If the CRE cannot be interpreted meaningfully within this context, we recursively attempt to resolve the CRE in the immediate parent of the current segment and then the parent's parent and so on, until we arrive at the right level for interpreting the CRE (Example 7). It is important to emphasize that the actual elements of a range cannot be deduced independently of the legal text because alphanumerics may be used in the numbering, as illustrated in Example 6. When multivalued CREs include implicit terms, we apply the same process as for simple CREs (see Example 4).

Interpreting multilayered CREs For multilayered CREs that do not contain multivalued CREs, interpretation is performed by harmonizing the navigation order so that it is strictly upward or downward. For example, all the following four CREs point to the same provision: (1) "article 131, 1st alinea, sub d)", (2) "sub d) of article 131, 1st alinea", (3) "1st alinea, sub d) of article 131", (4) "article 131 sub d) of 1st alinea". Only (1) is in a harmonized form. The harmonized form is easy to compute based on the topological order discussed in Section VI. The most complex form of CREs arises when layers are combined with ranges and enumerations. The regular expressions that detect such CREs are ambiguous.
To illustrate, consider the CRE in Example 8. Without knowing the structural makeup of the underlying text, one cannot know if the "127 and 154ter" fragment in
Example 8

$C R E$ : articles 109,1 st alinea, num bers 1 to 3, 127 and 154ter

Interp.: article 109 paragraph 1 number 1, article 109 paragraph 1 number $1 \mathrm{a}$, article 109 paragraph 1 number 2, article 109 paragraph 1 number 3 , article 127 , article 154 ter this CRE refers to articles, paragraphs, or numbers. One could take cues from punctuation and the singular vs. plural concept markers to rule out the fragment referring to paragraphs. One could further deduce that either 127 or 154ter has to be an article because the article concept marker is in plural form. Unfortunately, such reasoning is unreliable as punctuation and the use of singular vs. plural are not consistently followed in legal texts. For example, the distinction between singular and plural disappears when abbreviations (e.g., art.) are used.

We interpret multilayered CREs with ranges and enumerations in a similar manner to Example 7 . When faced with a CRE fragment whose type is unknown, an attempt is made to interpret that fragment in the deepest context previously used for interpretation. In the case of the CRE in Example 8, this means that first, we take the numerical expression "127" (whose type is unknown) to be the continuation of "numbers 1 to 3 ". An attempt is made to interpret "127" in the context of "article[s] 109,1 st alinea", i.e., the same context where "numbers 1 to 3 " was interpreted. After this attempt fails, we recursively switch to the upper-level context in the CRE, i.e., "article[s] 109" (the same context where "1st alinea" was interpreted). This second attempt also fails. The third attempt tries to interpret "127" in the context of all articles. This attempt succeeds. Now that "127" has been interpreted as an article, the CRE will be seen as if the concept marker article appeared just before "127" in the legal text. The remainder of the enumeration, i.e., "154ter", is interpreted as if the CRE is "articles 109, 1st alinea, numbers 1 to 3 , article 127 and 154ter".

\section{Linking Cross Reference Expressions to Target Provisions}

In the final step, we link each CRE to all the provisions resulting from its interpretation. Like the interpretation phase, the linkage phase requires knowledge of the hierarchy of the legal text in which the target provisions are located. The details of the linkage phase are straightforward. An example of how the resulting links are rendered is provided in Section VIII when discussing visualization and navigation.

\section{APPLICATIONS}

In this section, we outline some important use cases that build on the results of CR detection and resolution.

Visualization and Navigation. CR detection and resolution is a prerequisite for generating navigable views of legal texts. In Fig. 7, we show a small excerpt of an HTML view of Luxembourg's Income Tax Law. In this view, resolved CREs appear as hyperlinks. Clicking on a CRE brings up a tooltip box, allowing the user to navigate to any of the CRs entailed by the CRE. A view like this is useful during the elaboration of compliance requirements, where analysts often need to follow the CRs in search of additional relevant information. 


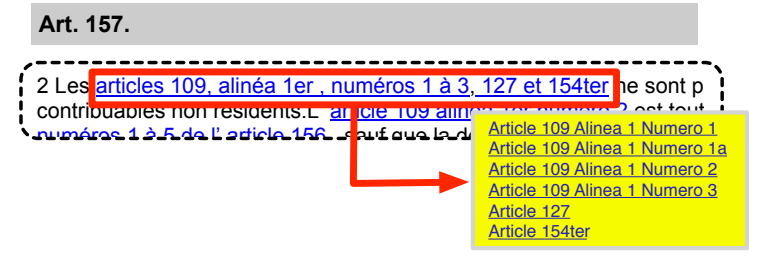

Fig. 7. A hyper-linked view of Luxembourg's Income Tax Law

Identifying Anomalous CREs. A natural byproduct of the resolution process are diagnostics about CREs that cannot be resolved. Failure to resolve a CRE may be due to it being nonwell-formed, or due to the CRE targets being non-existing. In either case, it is important for both legal experts and requirements analysts to be made aware of anomalous CREs.

Trace Link Analysis. Trace link analysis is concerned with identifying the set of provisions that refer to a particular provision. To illustrate the usefulness of this type of analysis, consider the following example from Luxembourg's Income Tax Law: "Art. 24" of this law elaborates the pension schemes recognized for taxation. A natural query for a requirements analyst who is elaborating the compliance requirements for taxation of pensioners is: Where is "Art. 24" referred to? A naive string search, e.g., "Art. 24", over the text of the law yields no results, despite the article being internally cited in four places. On all occasions, the article is cited within ranges: "Art. 4 to 155bis", "Art. 14 to 108bis", "Art. 16 to 60" and "Art. 106 to 60 ". Without automated support, identifying where the article is cited requires a manual scan of the entire law (189 pages).

To automate trace link analysis, we need a logical representation of the structure of the legal text(s) in question along with their CR links. This information is conveniently expressed as a typed graph [22] - intuitively, a graph whose nodes and edges are typed. In our problem, graph nodes represent instances of the grouping concepts in a legal text, e.g., individual chapters, sections, articles, and paragraphs. Each node type is thus one of the classes of the text schema. For Luxembourg's Income Tax Law, this is the schema of Fig. 3. Edges may represent two types of relationships: first, that a grouping concept instance contains another, e.g. Article 4 contains Article 4 Paragraph 2; and second, that a grouping concept instance (directly) cites another, e.g. Article 4 Paragraph 2 cites Article 46 Number 3.

Given such a logical representation, one can infer links between any pair of grouping concept instances. For example, one can identify all citations to a given article. We show the logical queries for this computation in the snippet of Fig. 8. The snippet is written in the Relational Manipulation Language (RML) - the query language for a first-order logic interpreter, named Crocopat [16]. In the snippet, we compute a relation, Contains $(\mathrm{x}, \mathrm{y})$, that holds for all $(x, y)$ where $y$ is a child of $x$ in the legal text's hierarchy tree. $\operatorname{TrContains}(\mathrm{x}, \mathrm{y})$ computes the reachability relations via containment using the transitive closure operator (TC). TrContains $(\mathrm{x}, \mathrm{y})$ thus holds for all $(x, y)$ where $y$ is a descendant of $x$. Cites $(\mathrm{x}, \mathrm{y})$ computes $(x, y)$ where $x$ directly cites $y$ via a CR. Finally, LinkedToArt( $(x, y)$ computes all $(x, y)$ where $y$ (i.e., the link target) is of type Article, and where $x$ cites some element $z$ that is transitively contained in $y$ (e.g., a paragraph of $y$, or a letter in a paragraph of $y$ ).

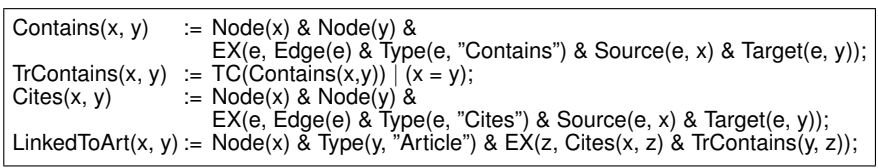

Fig. 8. Logical queries for trace link analysis

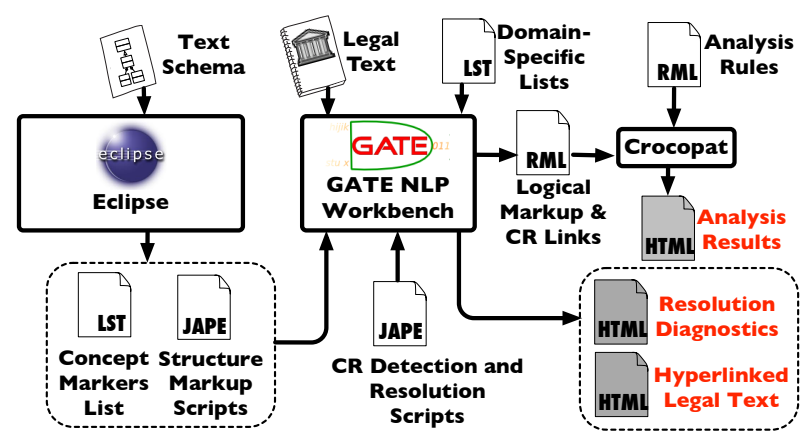

Fig. 9. LeCA tool architecture

Circularity Analysis. Cyclic citations are common in legal documents. A frequent usage is when a provision $X$ refers to a provision $Y$ to state that $X$ depends on $Y$ for a definition; and $Y$ refers back to $X$ to state that $Y$ provides a definition required by $X$. While cycles seldom indicate errors, they need to be investigated carefully to verify absence of circular reasoning, e.g., cases where provisions $X$ and $Y$ both depend on one another for a definition. Circularity analysis is performed using similar logical queries to what we illustrated in Fig. 8. We do not elaborate the queries due to space constraints.

\section{TOOL SUPPORT}

We implement our approach in a tool named LeCA (Legal Cross Reference Analyzer). LeCA provides automated support for (1) generation of text structure markup (Section VI), (2) CR detection and resolution (Section VII), and (3) visualization and CR analysis (Section VIII). LeCA builds on the GATE Workbench [15] - a mature open-source NLP framework.

Fig. 9 shows an overview of LeCA. Eclipse's model-to-text transformation facilities are used in order to derive, from a text schema, JAPE scripts for text structure markup. These scripts are then executed by GATE, followed by those for $\mathrm{CR}$ detection and resolution. The CR detection and resolution scripts rely on several keyword lists (gazetteers), e.g., concept markers, names of legal texts, and implicit terms. The list for concept markers is derived from the text schema. The remaining lists are domain-specific and depend on the language of the legal text and the specific jurisdiction to which the legal text belongs. These lists thus need to be provided by the user.

As output, LeCA produces an HTML view of the input legal text with CRs represented as hyperlinks. Diagnostics are provided for any unresolved CRs. LeCA additionally generates a logical representation of the input text's structure and CRs, which is in turn fed to a first-order logic interpreter, Crocopat [16], for analysis. Our analysis rules currently cover traceability link generation and identification of circular citation paths.

LeCA is written in GATE's regular expression language, JAPE (Java Annotation Patterns Engine). JAPE allows direct use of Java to specify the actions to take when a particular 
regular expression is recognized. This enables us to seamlessly integrate CR detection and resolution activities. In total, LeCA implements 113 JAPE scripts with approximately 10,000 lines of JAPE code, excluding comments and third-party libraries.

\section{Evaluation}

In this section, we describe an evaluation of our approach, aimed at answering the following Research Questions (RQs):

RQ1. Is our approach effective at detecting CREs? Our natural language patterns for CREs were gleaned from Luxembourg's Income Tax Law. RQ1 aims at verifying the completeness of the patterns by analyzing their accuracy for detecting CREs in legislative texts other than the Income Tax Law.

RQ2. Is our approach effective at resolving CREs? RQ2 aims at measuring how accurate our approach is in resolving CREs that have been already detected.

RQ3. Is our approach scalable? Legal texts can be hundreds and sometimes thousands of pages long. RQ3 aims at establishing whether our approach runs within reasonable time.

\section{Results and Discussion}

RQ1. To answer RQ1, we selected 13 legislative texts with a total of 1640 pages from Luxembourg's legal corpora. Our main selection criteria were to cover (1) a diverse set of texts, and (2) a large timespan in terms of when the texts were first drafted. The selected texts were drawn from civil laws, criminal and penal laws, social welfare, pensions, housing, commerce, and healthcare. The oldest text in our selection dates back to 1808 and the newest one to 2011 .

We randomly chose $10 \%$ of the pages in each selected text. If a randomly-chosen page coincided with the preface, table of contents, document history, or index, the page was discarded and another random page was considered. In total, we considered 164 pages of text containing actual legal provisions. We conducted a manual inspection of these pages and highlighted the CREs found. This inspection yielded 1852 CREs.

Following the inspection, we applied our tool for detecting the CREs in these pages. The tool was applied exclusively for detection, i.e., structural markup generation and resolution were not performed. For detection, we used the concept markers (line 4 of the patterns in Fig. 6) prescribed for legislative texts by Luxembourg's legal writing best practices that are in effect today [19]. For generic terms, law names and auxiliary terms (respectively, lines 7, 13 and 15 of the patterns), we exploited the lists built from our investigation of the Income Tax Law. Table II summarizes the results for RQ1. In the table, we classify the identified CREs across the same dimensions as those used in Table I for the Income Tax Law.

The results indicate that our patterns miss only one CRE amongst the ones investigated (less than one tenth of a percent). This CRE was at (1) (in French, au (1)), which referred to paragraph 1 of the article in question. The CRE can be detected by adding "at" to the generic terms (line 7 of the patterns). 38 CREs $(\approx 2 \%)$ were only partially identified. All cases were attributed to incompleteness in the lists of concept
TABLE II

RESULTS FOR RQ1

\begin{tabular}{|l|l|l|l|c|}
\hline CRE Type & \# of CREs & $\begin{array}{l}\text { Correctly } \\
\text { Identified }\end{array}$ & $\begin{array}{l}\text { Partially } \\
\text { Identified }\end{array}$ & Missed \\
\hline Internal & 857 & 848 & 8 & 1 \\
External & 995 & 965 & 30 & 0 \\
\hline Explicit & 1389 & 1350 & 38 & 1 \\
Implicit & 1852 & 463 & 0 & 0 \\
\hline Simple & 1031 & 1029 & 1 & 1 \\
Complex & 821 & 784 & 37 & 0 \\
- Mutlivalued & 373 & 372 & 1 & 0 \\
- Multilayered & 448 & 412 & 36 & 0 \\
\hline
\end{tabular}

markers, law names, and auxiliary terms. Detection further yielded 5 false positives (not shown in the table).

Without addressing the incompleteness in the lists, detection has a precision of $99.7 \%$, recall of $97.9 \%$ and $F$-measure of $98.8 \%$. If the lists are completed, these measures will respectively be: $99.7 \%, 100 \%$ and $99.8 \%$. No new patterns emerged from our investigation in RQ1, providing confidence about the completeness of our patterns for Luxembourg's legislation.

$\boldsymbol{R Q 2}$. We answer RQ2 based on the resolution of internal CREs in Luxembourg's Income Tax Law. Although this law is the same legal text from which we drew our CRE patterns, we believe that using this text towards answering RQ2 is justified in the light of the following: First, from our analysis of RQ1, one can be reasonably confident that our patterns achieve high coverage in detection. Second, our resolution algorithm is instantiated based on only the text schema and the CREs patterns, irrespectively of the actual legal text. We thus anticipate little bias resulting from using the Income Tax Law in RQ2. Our decision to use this law for resolution was necessitated by the fact that we had access only to legal experts in the area of taxation. Measuring the effectiveness of resolution requires a precise text schema, for which we needed to interact closely with legal experts. We further need to note that although our patterns address both internal and external CREs, our evaluation of RQ2 is exclusively concerned with internal ones. A detailed resolution of external CREs requires the text schema for the external legal texts. One could perform a coarse-grained resolution for external CREs by merely linking them to the target legal texts, as opposed to the specific provisions being cited within these texts. This is rather unfruitful from an evaluation standpoint.

We attempted automated resolution for all internal CREs except unspecific ones, i.e., a total of 883 CREs (see Table I in Section VII-A). This resulted in 1736 CR links plus 9 warnings for the CRs whose target provision could not be found. We then conducted a walkthrough of the resulting CR links and the warnings. All the 1736 automatically-generated links were deemed correct. Of the 9 warnings, 8 arose due to CREs that were indeed anomalous in the text of law. When combined, the CR links and the 8 warnings fully covered the internal CREs over which resolution was attempted.

The last warning was due to our algorithm incorrectly interpreting an external CRE as an internal one. The CRE in question is paragraph 11bis in the following excerpt: "The modified fiscal adjustment law of 16 October 1934 is amended with the following provision, inserted into the law as paragraph 11 bis: [...]". Since 
our approach does not analyze the semantics of sentences, it interprets paragraph 11 bis as being internal although the correct treatment is paragraph 11 bis of the law of 16 October 1934 .

With regards to the accuracy of resolution over the Income Tax Law, we get a precision of $99.9 \%$, recall of $100 \%$, and $F$ measure of $99.9 \%$ when we exclude unspecific CREs. From a practical standpoint, since we rely on a human expert to resolve the unspecific CREs, it seems more reasonable to treat these CREs as false negatives. This treatment provide a better reflection of the amount of manual work saved by automated resolution. Under this treatment, we obtain a precision of $99.9 \%$, a recall of $97.5 \%$, and $F$-measure of $98.7 \%$.

$\boldsymbol{R Q 3 . ~ T h e ~ e x e c u t i o n ~ t i m e ~ o f ~ o u r ~ a p p r o a c h ~ i s ~ d o m i n a t e d ~ b y ~}$ the resolution phase. For the Income Tax Law, interpreting CREs into individual $\mathrm{CRs}$ took $\approx 151$ seconds. Linking the CRs to the target provisions took a further $\approx 139$ seconds. The interpretation step has embedded into it the time spent for transforming the Income Tax Laws from plain text to XML. The execution time associated with the identification of CREs using regular expressions was $\approx 15$ seconds for the Income Tax Law and $\approx 34$ seconds for the selected pages in RQ1. Execution times were measured on a standard laptop with a 2.3 $\mathrm{GHz}$ Intel CPU and 8GB of memory. Given the short overall running time of our approach, we expect it to be scalable to larger legal corpora with thousands of pages.

\section{CONCLUSION}

We presented an approach for automatic detection and resolution of cross references in legal texts. Our approach complements existing work in a number of ways. In particular, the approach is parameterized by a text schema, making it possible to tailor the approach to different legal texts and jurisdictions. This text schema further allowed us to automatically enhance non-markup legal texts with the structural markup that is necessary for resolving cross references. Through a study of legislative texts in Luxembourg, we extended existing natural language patterns for cross reference expressions and provided a systematic way to interpret these expressions. We outlined the implementation of our approach in a Natural Language Processing environment. Our evaluation of the approach indicates that it is scalable and accurate in detecting and resolving cross references in Luxembourg's legislation.

Our work in this paper is part of a collaborative effort with the Government of Luxembourg on legal compliance in eGovernment. An important challenge in this context is managing the continuous evolution of government IT systems as well as the laws and regulations that apply to these systems. Cross references provide important cues not only about the relationships between legal provisions but also about the relationships between the compliance requirements derived from these provisions. Consequently proper handling of cross references is essential for automated traceability and change analysis over compliance requirements.

In future work, we plan to conduct larger-scale evaluations of our approach and analyze the effectiveness of resolution for external cross references. We would further like to investigate how changes in legal provisions bring about changes in compliance requirements. Another interesting topic for future work is to generalize our approach beyond legislative texts, and to regulations, circular letters, and parliamentary proceedings. Being able to automatically link and navigate these different types of legal texts makes it easier to analyze the rationale behind compliance requirements and the way they need to be operationalized.

Acknowledgments. Financial support for this work was provided by Luxembourg's National Centre of Information Technologies (CTIE) and Luxembourg's National Research Fund (FNR) under grant number FNR/P10/03. We are grateful to members of Luxembourg Inland Revenue Office (ACD) and CTIE, particularly, Thierry Prommenschenkel, Ludwig Balmer, Marc Blau, and Michael Masseroni for sharing their valuable knowledge and insights with us.

\section{REFERENCES}

[1] Govt. of Luxembourg, "Modified Law of Dec. 4, 1967 (Income Tax) (Loi modifiée du 4 déc 1967 concernant l'impôt sur le revenu)," 2013.

[2] J. Maxwell and A. Antón, "The production rule framework: Developing a canonical set of software requirements for compliance with law," in 1st ACM Intl. Health Informatics Symp., 2010, pp. 629-636.

[3] T. Breaux and A. Antón, "Analyzing regulatory rules for privacy and security requirements," IEEE TSE, vol. 34, no. 1, pp. 5-20, Jan. 2008.

[4] T. Breaux, A. Antón, K. Boucher, and M. Dorfman, "Legal requirements, compliance and practice: An industry case study in accessibility," in $R E$, 2008, pp. 43-52.

[5] J. Maxwell, A. Antón, P. Swire, M. Riaz, and C. McCraw, "A legal crossreferences taxonomy for reasoning about compliance requirements," REJ, vol. 17, no. 2, pp. 99-115, Jun. 2012.

[6] J. Maxwell, A. Antón, and J. Earp, "An empirical investigation of software engineers' ability to classify legal cross-references," in $R E$, 2013, pp. 24-31.

[7] S. Ghanavati, "Legal-URN framework for legal compliance of business processes," Ph.D. dissertation, 2013.

[8] R. Hoekstra, "The metalex document server," in 10th Intl. Conf. on The Semantic Web, 2011, pp. 128-143.

[9] T. Breaux, "Legal requirements acquisition for the specification of legally compliant information systems," Ph.D. dissertation, 2009.

[10] M. Palmirani, R. Brighi, and M. Massini, "Automated extraction of normative references in legal texts," in 9th Intl. Conf. on AI and Law, 2003, pp. 105-106.

[11] E. de Maat, R. Winkels, and T. van Engers, "Automated detection of reference structures in law," in 19th Annual Conf. on Legal Knowledge and Information Systems, 2006, pp. 41-50.

[12] M. Hamdaqa and A. Hamou-Lhadj, "An approach based on citation analysis to support effective handling of regulatory compliance," Future Generation Computer Systems, vol. 27, no. 4, pp. 395-410, 2011.

[13] O. Tran, M. Le Nguyen, and A. Shimazu, "Reference resolution in legal texts," in 14th Intl. Conf. on AI and Law, 2013, pp. 101-110.

[14] The Bluebook: A Uniform System of Citation, 19th ed. Harvard Law Review, 2010.

[15] Cunningham et al, "Developing Language Processing Components with GATE Version 7 (a User Guide)." [Online]. Available: http://gate.ac.uk

[16] D. Beyer, A. Noack, and C. Lewerentz, "Efficient relational calculation for software analysis," IEEE TSE, vol. 31, no. 2, pp. 137-149, 2005.

[17] A. Massey, P. Otto, and A. Antón, "Prioritizing legal requirements," in RELAW, 2009, pp. 27-32.

[18] Association of Legal Writing Directors and D. Dickerson, The ALWD Citation Manual, 4th ed. Aspen Publishers, 2010.

[19] M. Besch, "Traité de légistique formelle," 2005.

[20] T. Cormen, C. Leiserson, R. Rivest, and C. Stein, Introduction to Algorithms, 3rd ed. MIT Press, 2009.

[21] J. Levine, T. Mason, and D. Brown, Lex \& Yacc. O'Reilly, 1992.

[22] G. Rozenberg, Ed., Handbook of graph grammars and computing by graph transformation (Vol. 1): Foundations. World Scientific, 1997. 